\title{
BMJ Open Parental engagement and early interactions with preterm infants during the stay in the neonatal intensive care unit: protocol of a mixed-method and longitudinal study
}

\author{
Alberto Stefana, Manuela Lavelli
}

To cite: Stefana A, Lavelli M. Parental engagement and early interactions with preterm infants during the stay in the neonatal intensive care unit: protocol of a mixed-method

and longitudinal study. BMJ Open 2017;7:e013824. doi:10.1136/bmjopen-2016013824

- Prepublication history for this paper is available online. To view these files please visit the journal online (http://dx.doi.org/10.1136/ bmjopen-2016-013824).

Received 10 August 2016 Revised 7 November 2016 Accepted 2 December 2016

CrossMark

Department of Human Sciences, University of Verona, Verona, Italy

Correspondence to Alberto Stefana; alberto.stefana@email.it

\section{ABSTRACT}

Introduction: The preterm infants' developmental outcomes depend on biological and environmental risk factors. The environmental factors include prolonged parental separation, less exposure to early mother/ father-infant interactions and the parents' ability to respond to the trauma of premature birth. In the case of premature birth, the father's ability to take an active part in the care of the infant from the start is essential. The parents' emotional closeness to the preterm infant hospitalised in the neonatal intensive care unit (NICU) may be crucial to the well-being of the newborn, the development of mutual regulation, the establishment of a functioning parent-infant affective relationship and the parents' confidence in their ability to provide care for their baby.

Methods and analysis: This is a mixed-method, observational and longitudinal study. The methodological strategy will include: (1) ethnographic observation in a level III NICU located in Italy for a duration of 18 months; (2) 3-minute video recordings of mother-infant and father-infant interaction in the NICU; (3) a semistructured interview with fathers during the infants' hospital stay; (4) 3-minute video recordings of mother-infant and father-infant face-toface interaction in the laboratory at 4 months of corrected age; (5) self-report questionnaires for parents on depression and quality of the couple relationship at the approximate times of the video recording sessions.

Ethics and dissemination: The study protocol was approved by the Ethical Committee for Clinical Trials of the Verona and Rovigo Provinces. Results aim to be published in international peer-reviewed journals, and presented at relevant national and international conferences. This research project will develop research relevant to (1) the quality and modalities of maternal and paternal communication with the preterm infant in the NICU; (2) the influence of maternal/paternal social stimulation on the infant behavioural states; (3) the quality and modalities of paternal support to the partner and possible influences on mother-infant relationship.

\section{Strengths and limitations of this study}

- This is one of a small number of studies focused on maternal/paternal communicative behaviours addressed spontaneously to the preterm infant hospitalised in the neonatal intensive care unit (NICU), and their effects on the infant's behavioural states.

- Results from this project will increase the very scant knowledge about the presence of early interactive contingencies between mother/father and the preterm infant in the NICU, and their possible predictive role of positive outcomes in mother-infant and father-infant relationship.

- Findings will be limited to the experiences of Italian parents.

\section{INTRODUCTION}

The preterm birth is a physiologically traumatic event in which infants' healthy neurological and emotional development is threatened. ${ }^{1-5}$ The preterm infants' developmental outcomes depend on biological and environmental risk factors. ${ }^{6-10}$ During the period of hospitalisation in the neonatal intensive care unit (NICU), environmental factors include physical (eg, excessive noise and light levels, painful procedures) and psychosocial (eg, prolonged parental separation) stressors. ${ }^{11}$ Furthermore, preterm infants in incubators cannot experience the earliest mother-infant interactions which play a crucial role in early regulation of the stress response ${ }^{1314}$ and provide the foundations for the development of mutual regulation. ${ }^{15}$ These skills are known to have a long-term impact on the functioning of affective relationships and healthy developmental outcomes. ${ }^{6}$ The immature brain of a preterm infant is particularly vulnerable to the quality of these experiences. ${ }^{16}$ 
When a baby is born prior to the physiological term of 39 weeks, the process of preparation for parenthood is also interrupted ahead of time. ${ }^{17}{ }^{18}$ In this way, parents are also premature. ${ }^{19}$ In this situation, parents are required to adjust their emotional structures in order to respond to the trauma of premature birth. ${ }^{20}{ }^{21}$ This process is exceptionally important as the inability to respond to the trauma may have negative consequences on infant development. ${ }^{22} 23$ Often, mothers require a recovery period after the premature birth. As a result, the father's ability to take an active part in the care of the infant from birth may be crucial. ${ }^{24}$ However, fathers can face many obstacles that can impact on their roles as partners and fathers,${ }^{25-27}$ with possible negative consequences for their partners' affective states (eg, depression, anxiety), ${ }^{28-31}$ and for the development of parental relationships with the infants. ${ }^{29} 32$ Furthermore, the birth of a preterm infant might have a negative impact on self-representations of fathers during the child's stay in the NICU. Therefore, in order to adequately sustain fathers in their involvement in the infants' care, and in their transition to parenthood, specific supports are required from the NICU staff. ${ }^{33}$ To date, however, only a few studies have focused their investigations on the father's role in preterm birth. ${ }^{34} 35$

Past and recent studies exploring the quality of care given to preterm infants in the NICUs have shown that parent-preterm infant closeness during hospitalisation, particularly skin-to-skin contact with the mother, and interventions aimed at supporting parental involvement in infant care may be crucial to the well-being of the newborn (enhancing neurobehavioural outcomes), ${ }^{36-41}$ the establishment of parent-infant relationship ${ }^{42}$ and the parents' sense of confidence in providing care for their baby. ${ }^{38}$ Other recent studies have shown that exposure to recorded or live maternal/parental voice has beneficial effects on physiological and behavioural states of preterm infants, ${ }^{43-45}$ and predicts infants' vocalisations more than the voices of other adults. ${ }^{46}$ However, very few studies have focused on mothers, ${ }^{47-49}$ or both parents, ${ }^{29}$ spontaneous behaviours with their infants in the NICU: They have observed caregiving routines before discharge, when the baby was allowed to spend some time out of the incubator. To the best of our knowledge, only one study ${ }^{50}$ has been conducted on maternal behaviours addressed to the preterm infant hospitalised in the NICU, particularly when the infant was distressed, and on how the maternally mediated sensory experience of the infant may impact the infant's behavioural states and mother-infant interaction. The present study expands the focus to maternal and paternal communicative behaviours addressed spontaneously to the preterm infant in a heated cot in the NICU.

It is important to acknowledge that parents of preterm infants are at great risk of psychological distress and depressive symptoms ${ }^{28} 5152$ that can interfere with their sensitivity to infant cues. ${ }^{29}{ }^{53}$ For instance, it has been found that mothers who experience traumatic stress in the perinatal period tend to be less sensitive and more controlling at 6 months of infant's corrected age (CA), ${ }^{50}$ and that parental depression-as an inhibitory factor of good parent-infant interactions-has a negative impact on the early neurobehavioural ${ }^{54}$ and socioemotional ${ }^{55}$ development of the infant. Some studies focused on mother-preterm infant interaction have shown that mothers of preterm infants tend to look, vocalise and touch their infants affectionately less often than mothers of full-term infants; in addition, preterm infants tend to spend less time in alert state, to be more passive as social partners and to send more unclear communicative signs, so that parent-infant interactions are less coregulated than with full-term infants ${ }^{48} 5657$ and a scant dyadic synchrony persists at 12 months of $\mathrm{CA}^{58}$ However, studies $^{40} \quad 56-62$ focused on mother-preterm infant interaction have also shown inconsistent findings ${ }^{61}$ though part of this discrepancy could be explained by the use of different observational methods and techniques (eg, rating scales vs microanalytic coding systems). Moreover, almost all these studies have looked at interactive and socioemotional behaviours after discharge from the NICU, over the infant's first 2 years (usually at 3 or 6, 12, 18 and/or 24 months of CA). And yet, to the best of our knowledge, no study has microanalytically coded mother-infant and father-infant interactions during a stay in a level III NICU.

\section{Objectives}

In light of the above, the first objective of this study is to examine maternal and paternal communication with their preterm infant in a heated cot in the NICU, analysing the presence of interactive contingency between parental communicative behaviours and infant gaze direction and expression indexing the infant's engagement in the interaction. 'Interactive contingency' is defined as the predictability of each partner's behaviour from that of the other, over time. ${ }^{63} 64$

A second objective, consequent on the first, is to assess whether early interactive contingency between mother/ father and preterm infant in the NICU predicts positive outcomes in the mother-infant/father-infant relationship and infant development at 4 months CA.

A third objective, parallel to the first, is to investigate the emotional impact of the premature birth on parents, particularly on fathers. This objective will be accomplished by examining a number of different factors, namely, the ways through which the fathers' perception of their parental role (or absence of role) influences their engagement in caring the baby, their support to the partner and the mother-infant relationship.

Finally, the last and overarching objective is to examine the relationship between (1) maternal and paternal emotional conditions after the premature birth, (2) the infant perinatal risk factors and (3) the quality of mother-infant and father-infant interactions during the stay in the NICU, and later, at 4 months CA. 


\section{METHOD AND ANALYSIS}

This is a mixed-method, observational and longitudinal study. The methodological strategy will include: (1) ethnographic observation in a level III NICU located in Northern Italy for the duration of data collection over an 18-month period; (2) one 3-minute video recording of mother-infant and father-infant face-to-face interaction in the NICU (with the preterm infant in a heated cot) between 34 and $35+6$ weeks postmenstrual age (PMA); (3) a semistructured interview with the fathers during the infants' stay in the NICU; (4) one 3-minute video recording of mother-infant and father-infant face-to-face interaction in the Social and Language Development Laboratory, University of Verona, at 4 months CA; (5) a self-report questionnaire on depression and a questionnaire on the quality of the couple relationship submitted to the parents at the approximate times of the video recording sessions.

\section{Selection criteria}

\section{Inclusion criteria}

Infants are eligible to be included in the study if they meet the criterion of: (1) birth before 34 weeks PMA. The reason for choosing a cut-off of 34 weeks (instead, for instance, 32 weeks, ie, the cut-off for very preterm birth) is to increase the number of participant families, given the difficulties of recruitment in the NICU. Furthermore, this criterion is in line with other studies on maternal caregiving behaviour during the stay in the NICU, ${ }^{50}$ and on mother-preterm infant interaction after discharge. ${ }^{5765}$

Mothers and fathers are eligible to be included in the study if they meet the following criteria: (1) both were born and grew up in Italy; (2) both have given their consent to participate in the study.

\section{Exclusion criteria}

Infants will be excluded from the study if: (1) they have perinatal asphyxia; (2) they have neurologic pathologies (periventricular leucomalacia up to stage I and/or intraventricular haemorrhage up to stage II); (3) they experience malformation syndromes and/or major malformations; (4) they have sensory deficits (detected by regular medical checks performed during hospitalisation); (5) they present metabolic or genetic disease.

Mothers and fathers will be excluded from the study if: (1) they are not of Italian origin; (2) they have a psychiatric illness; (3) they have issues with drug or substance abuse; (4) they are not living together; (5) they are adopting parents; (6) they are a same-sex couple.

To participate in the study it is necessary that both parents and infants meet the inclusion and exclusion criteria.

\section{Procedure}

The timeline of the whole procedure, beginning with ethnographic observation even before the participants' recruitment, is depicted in figure 1.

\section{Ethnographic observation in NICU}

Ethnography is a methodological approach which involves the researcher participating overtly in the lives of people, in this case in the NICU. ${ }^{66}$ Ethnographers work by 'watching what happens, listening to what is said and/or asking questions through informal and formal interviews and collecting documents' ${ }^{68}$ Ethnographic observation was selected over other methods to inform this study and to prepare the best conditions for accomplishing this study, as it aims (1) to minimise the risks potentially associated with having an observing researcher within the observed social context (eg, perception of intrusiveness, minor spontaneity during videotaped interactions and/or interviews) and (2) to obtain rich qualitative data from participant observation in social interactions which take place in that context. This is even more important in a stressful context such as the NICU, where social interactions are particularly vulnerable.

Initially, the researcher (the psychologist-psychotherapist and $\mathrm{PhD}$ student who is the first author) goes through a familiarisation period with the parents in the NICU. During this period and later on too, the researcher observes the interactions between (1) parents and their preterm infants, (2) mothers and fathers, (3) parents and staff (nurses, neonatologist and psychologist) and (4) infant and nurses; he attends meetings between
Figure 1 Study timeline. $\mathrm{CA}$, corrected age; PMA, postmenstrual age; wk, weeks.

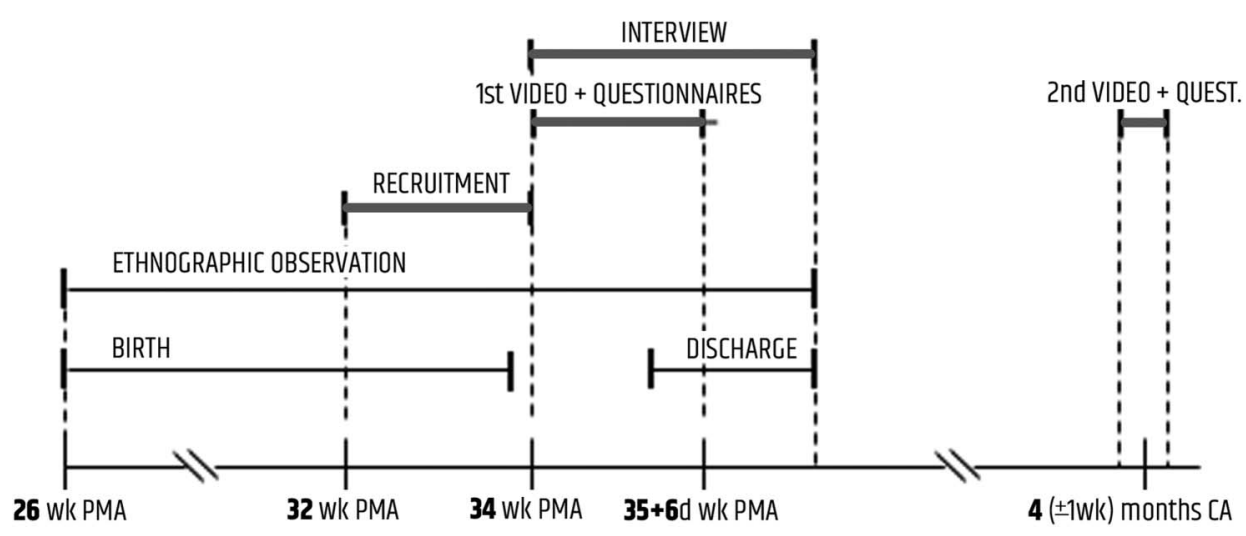


neonatologists, observes conversations between staff and parents and he also holds informal talks with mothers and fathers. Then the researcher presents the research project to the parents who meet the inclusion criteria and 'follows' the recruited families until the infant's discharge from the NICU. We think that qualitative data obtained from ethnographic observation can offer a main contribution to the understanding of the NICU-related stress and the impact of preterm birth and ensuing hospitalisation on mothers' and fathers' emotional experiences, their relationship and how they cope with the stay in the NICU: all factors that might affect parent-infant relationship and the quality of motherinfant and father-infant interactions. Finally, ethnographic observation is used to identify the best conditions -infant's behavioural states, time, position and so onfor video recording parent-infant face-to-face interaction (with the preterm infant in a heated cot) in the NICU.

\section{Recruitment}

All parents (mothers and fathers) of preterm infants born between 17 September 2015 and 31 March 2017 and hospitalised at level III NICU of the Borgo Roma Hospital-Azienda Ospedaliera Universitaria Integrata Verona (northern Italy) are invited to participate in the study. The researcher invites the parents to participate during the stay in the NICU, when the infant's medical condition has been stabilised: this usually happens around or after 32-33 weeks PMA; therefore, the recruitment of the study participants takes place from 32 to 34 +6 weeks PMA. A minimum of 20 mother-infant and 20 father-infant dyads will be recruited. This sample size is adequate to perform parametric statistical tests, and is in line with the sample size of previous Italian studies ${ }^{47586970}$ in the field.

\section{Parent-infant interaction in NICU}

Between 34 and 35+6 weeks PMA, the mother's spontaneous social stimulation addressed to the infant in a cot with a radiant heater (an open cot with an overhead heating source) and the infant's responses are videotaped during a 3-minute interaction. Then the infant rests for at least $5 \mathrm{~min}$. Afterwards, the father's social stimulation addressed to the infant and the latter's responses are videotaped over a 3-minute interaction. The date and timing for video-recording parent-infant interaction depends on: (1) the infant's medical conditions, (2) the mother's and father's restrictions on the time available to stay in the NICU, (3) the infant's behavioural states (for instance, if the infant is sleeping or crying the video recording is postponed).

\section{Qualitative interviews in NICU}

Previous studies that have conducted interviews with fathers of premature infants provide a coherent picture. The preterm birth of an infant is a traumatic event for the fathers, ${ }^{71}$ who have been found to show low/moderate levels of adjustment to preterm birth and a limited assumption of paternal role. ${ }^{72}$ Research also suggests that fathers experience a sense of lack of control. ${ }^{73}$ Often fathers hide these emotional difficulties from healthcare providers, ${ }^{25}{ }^{74}$ but reported a need to share ${ }^{70}$ with someone who can understand. ${ }^{24}$ The first moments with the preterm infants evoke a 'rollercoaster of emotions ${ }^{, 75}$ which can lead fathers to become emotionally, and sometimes physically, distanced from the situation. After the initial shock and trauma, research has shown that fathers can experience emotional closeness ${ }^{27}$ with their infants during hospitalisation in the NICU.

Before the infant's discharge from the NICU, fathers are contacted to participate in a semistructured interview. The interviews allow for a deeper understanding of the emotional impact that the premature birth has on fathers. The topic guide includes the following areas: the first time that the father saw and/or touched his baby; the bond with the baby; caregiving activities; feelings associated with seeing the partner/mother care for, feed or cuddle their baby; space for one's own emotions when supporting and assisting the partner; work and management of their responsibilities in the outside world during the period of hospitalisation in NICU; the quality of the couple relationship during pregnancy and the period of hospitalisation in NICU; and how their feelings towards the baby change from his/her preterm birth to the time of discharge from the NICU.

In addition to the fathers who will participate in the parent-infant interaction, we will recruit an additional number of fathers who meet the inclusion criteria for parents and infants. These additional participants will not need to meet the infants' exclusion criteria. This will allow the study to have a more representative sample of daily clinical reality.

Interviews are carried out in a private room in the NICU, and are digitally recorded.

\section{Questionnaires in NICU}

In approximate times with videotaping, parents are asked to complete the Center for Epidemiologic Studies Depression Scale (CES-D $)^{76}$ to assess symptoms of depression and the Dyadic Adjustment Scale (DAS) ${ }^{77}$ to assess the quality of the couple's relationship.

\section{Parent-infant interaction after discharge}

At 4 months CA, 3 min of mother-infant and $3 \mathrm{~min}$ of father-infant spontaneous face-to-face interaction are videotaped in the Social and Language Development Laboratory, University of Verona. The infant is seated in an infant seat on a table; the mother/father, seated opposite the infant, is asked to play with the baby as she/he would at home. Two video cameras are used to generate a split-screen view of the interaction.

\section{Questionnaires after discharge}

Parents are asked to complete the CES-D Scale and the DAS Scale again at 4 months CA, in order to assess symptoms of depression and the quality of the couple's 
relationship around 5 months after discharge from the NICU.

\section{Further data on parents and infants}

Data are collected on the parents' demographic and socioeconomic characteristics (age, marital status, years of formal education, occupation, family composition and social network), on the mothers' gynaecological and obstetric history, on their infant's neonatal characteristics (gestational age, weight, height and head circumference) and on the severity of the perinatal problems (evaluated using the Perinatal Risk Inventory; PERI ${ }^{78}$ will be collected. Information are also collected regarding the infant's hospitalisation and developmental outcomes (assessed after discharge in a follow-up based on a paediatric examination including a somatic and neurological status assessment).

\section{Data analysis}

Video coding

Mother/father's and infant's behaviours from videotaped interactions are coded microanalytically, using units of $1 \mathrm{~s} .{ }^{79-81}$ Parent and infant behaviours are coded independently in ordinalised scales required for performing time series analysis (see below).

With regard to parent-infant interactions in the NICU, both parent and infant behaviours are coded in composite categories of Engagement Scales, recently devised by Lavelli and Beebe: ${ }^{82}$ the Mother/Father Engagement Scale, ordinalised from a high of maternal affiliative behaviour ${ }^{45}$ (composed of 'gaze at infant face + affectionate or static touch + affectionate talk and/or positive facial affect') to a low of 'gaze off'; and the Infant Engagement Scale, ordinalised from high levels of 'gaze at parent face + smile' and 'gaze at parent face + active movements' to a low of 'negative expression'. Communicative modalities included in maternal affiliative behaviour have been described as the main components of the maternal postpartum repertoire in humans and as predictor of positive outcomes. ${ }^{48}$ Among these modalities, in the NICU context maternal/paternal static touch as firm and sustained touch ${ }^{47}$ is an effective and salient way to be in contact with the preterm infant, given the loss of physical contact with the mother and the prolonged separation that results from the NICU experience; therefore, otherwise that with full-term infants, static touch is not considered less optimal than affectionate touch.

With regard to parent-infant face-to-face interaction at 4 months CA, parents' behaviours are coded according to their gaze direction (on-off the infant's face); mother/ father touch, ordinalised from a high of 'affectionate' to a low of 'intrusive' using the Maternal Touch Scale ${ }^{83}$ adapted to the Italian caregiving culture; mother/father facial affect, based on Beebe $e t a l^{81}$ and ordinalised from a high of 'mock surprise' to a low of 'negative'. Infants' behaviours are coded according to their gaze direction (on-off the infant's face); infant vocal affect, adapted from Beebe $e t a l^{81}$ and ordinalised from 'high positive' to 'angry protest/cry'; infant facial affect, adapted from Beebe et $a l^{11}$ and Lavelli and Fogel, ${ }^{15}$ and ordinalised from 'high positive' to 'negative'.

The inter-rater reliability (Cohen's $\kappa$ ) for maternal/ paternal/infant behaviours will be calculated on $20 \%$ of the videotapes, which will be coded by a second researcher who is blind to the aims of the study.

\section{Quantitative data analysis}

To explore the possible presence of early interactive contingency between parental communicative behaviours and infant gaze direction and expression (objective 1), a sequential analysis (conditional probabilities, GSEQ software) ${ }^{84}$ will be computed to examine the probabilities that the infant gazes at the parent's face with positive/neutral expression when the mother/ father provides affiliative behaviour, and vice versa, that the mother/father provides affiliative behaviour when the infant shows to be engaged or ready for social stimulation. Furthermore, since the conditional probabilities analysis highlights specific associations of behaviours but not how any interactive contingency unfolds during the entire segment of videotaped interaction, a time-series modelling ${ }^{83}$ of the moment-to-moment sequence of behaviours will be performed to explore the parents and infants capability to coordinate their behaviours at any level of engagement versus disengagement across the videotaped session. Self-contingency will be computed as exploratory variable of the timeseries analysis.

With regard to the second objective, that is, to assess whether higher engagement coordination (interactive contingency) between mother/father and preterm infant in the NICU predicts higher engagement coordination (interactive contingency) during parent-infant face-to-face interaction at 4 months CA, a multilevel time-series analysis ${ }^{83}$ will be performed. This analysis will allow to create indices of self-contingency (autocorrelation) and interactive contingency (cross-correlation) using the different possible pairs of parent-infant communication modality coded in behavioural scale (eg, infant gaze-mother/father gaze; infant vocal affectmother/father touch, etc): these results will shed light on the process of mutual regulation during interaction at 4 months CA. Then, to assess the hypothesis that interactive contingency between parent and infant in the NICU is longitudinally related to the quality of their relationship at 4 months CA, it will be necessary to create an Infant Engagement Scale at 4 months, through an algorithm, as well as a Mother/Father Engagement Scale from their interaction at 4 months, in order to have behavioural scales which are comparable with those used for coding interaction in the NICU.

Finally, the hypothesis of early interactive contingency as a predictor of later mutual engagement will be also assessed by a linear regression analysis. A set of linear regression analyses will be performed using different 
study variables as possible predictors of quality in mother-infant and father-infant interactions at 4 months $\mathrm{CA}$, in order to contribute to investigate the relationship between the different variables (objective 4)

\section{Qualitative data analysis, and following mixed analyses}

A thematic content analysis ${ }^{85} 86$ of the fathers' interviews will allow us to investigate the emotional impact of the premature birth on fathers (objective 3). To this aim, results will be integrated with field notes taken during ethnographic observation and results from the self-report instrument assessing symptoms of depression. All transcripts will be verified by one researcher before data analysis by listening to the audio recording and checking for accuracy of the written transcript. The transcripts will be analysed using a thematic content analysis for each question in the interview. Thematic analysis is a qualitative method for identifying, analysing and reporting themes within data. ${ }^{87}$ Transcripts will be read and reread so as to become familiarised with the data. Data will be managed using NVivo 11 (QSR International, USA). The first author will use an initial open coding to allow for the emergence of recurrent themes across fathers. Response patterns that will be relevant across all fathers will be coded and organised in thematic categories (along with verbatim quotes which illustrate each theme) ${ }^{87} 88$ All codes will be compared and contrasted, and then examined and discussed by the first author and two other researchers to identify meaningful categories or emergent themes. Codes with a single occurrence will be removed. Coding reliability and face validity achieved by remaining codes will be checked. A final coding scheme (defining each theme with verbatim quotes) will be collated. All transcripts will be recoded by the first author using the coding scheme. The inter-rater reliability will be calculated on $20 \%$ of the transcriptions, which will be coded by a second researcher.

A bivariate correlation analysis between theme frequencies emerging from the fathers' interviews, the scores questionnaires indexing symptoms of maternal/ paternal depression and the quality of the couple relationship, the scores from the PERI ${ }^{78}$ and behavioural measures of quality in parent-infant interaction such as maternal/paternal affiliative behaviour, early interactive contingencies and mutual engagement at 4 months, will be performed to assess possible concurrent and predictive associations, and patterns of association, between study variables (objective 4 ).

Qualitative data will contribute to explain results from statistical analyses.

\section{ETHICS AND DISSEMINATION}

\section{Description of risks}

There will be no risks associated with participation in any aspect of the described study.

\section{Informed consent}

Before taking part in the study all mothers and fathers will receive written and oral information about the content and extent of the study. If they are willing to participate, they will sign the informed consent form. They will be able to withdraw from the study at any time without explanation, without any consequences to the care of the family at the Azienda Ospedaliera Universitaria Integrata Verona.

\section{Data protection}

All the video files, coding sheets, audio files, audio transcriptions, questionnaires and documents, will be provided with a special alphanumeric code and will not contain any identifying information. The electronic data will be stored on a computer that is password protected. The paper materials will be stored in a locked archive. Only members of the study team will have access to the data.

\section{Scientific, clinical and social impact}

To the best of our knowledge, this is the first research project focused on maternal/paternal communicative behaviours addressed spontaneously to the preterm infant hospitalised in the NICU, and their effects on the infant's behaviours and engagement in social interaction. Therefore, this research project will provide new knowledge in the particular area of: (1) the quality and modalities of maternal and paternal communication with the preterm infant in the NICU when the infant is in a cot with a radiant heater; (2) the influence of maternal/paternal social stimulation on infant's behavioural states, and the associated potential benefits for the preterm infant; (3) the quality and modalities of paternal support to the mother/partner, and the ways in which this influences the mother-infant relationship. In addition, results from this project will increase the very scant knowledge about the presence of early interactive contingencies between mother/father and preterm infant in the NICU, and their possible predictive role of positive outcomes in mother-infant and father-infant relationship.

Knowing more about under what conditions preterm infants hospitalised in the NICU could benefit from parental social stimulation has important clinical implications that could inform nursing practice and psychological support. First, this knowledge highlights possible factors on which early intervention programmes in the NICU should focus in order to support the development of healthy mother-infant, father-infant and mother-father relationships. Second, analysing the quality of maternal/paternal communication with the preterm infant in a heated cot allow for the identification of early indices of risk in the developing mother/ father-preterm infant relationship. This may in turn help to improve the identification of mother/fatherinfant dyads who are at-risk and who might benefit from early preventive intervention. With regard to this, staff 
members in the NICU might become more aware of individual differences in mothers and fathers that could facilitate or interfere with the parents' ability to provide sensitive care for their preterm infants.

\section{DISSEMINATION}

The results of this study will be submitted for publication in international peer-reviewed journals. Additionally, key results will be presented at relevant national and international conferences. Finally, the results of this study will part of the first author's PhD thesis

Contributors AS was involved in the general study design as $\mathrm{PhD}$ research project. ML served primarily as research project supervisor. AS focused on and developed the qualitative aspects of the study protocol. ML designed the coding system and the plan of statistical analysis of the study protocol. AS and $\mathrm{ML}$ shared the writing of the manuscript.

Competing interests None declared.

Patient consent Obtained.

Ethics approval Ethical approval of the study protocol was obtained from the Ethical Committee for Clinical Trials of the Verona and Rovigo Provinces (reference no. 569CESC).

Provenance and peer review Not commissioned; externally peer reviewed.

Data sharing statement It is a study protocol. All data will be published.

Open Access This is an Open Access article distributed in accordance with the Creative Commons Attribution Non Commercial (CC BY-NC 4.0) license, which permits others to distribute, remix, adapt, build upon this work noncommercially, and license their derivative works on different terms, provided the original work is properly cited and the use is non-commercial. See: http:// creativecommons.org/licenses/by-nc/4.0/

\section{REFERENCES}

1. Doyle LW, Anderson PJ. Adult outcome of extremely preterm infants. Pediatrics 2010;126:342-51.

2. Johnson S, Fawke J, Hennessy E, et al. Neurodevelopmental disability through 11 years of age in children born before 26 weeks of gestation. Pediatrics 2009;124(2).

3. Marlow N, Wolke D, Bracewell MA, et al. Neurologic and developmental disability at six years of age after extremely preterm birth. N Engl J Med 2005;352:9-19.

4. Mwaniki MK, Atieno M, Lawn JE, et al. Long-term neurodevelopmental outcomes after intrauterine and neonatal insults: a systematic review. Lancet 2012;379:445-52.

5. Talge NM, Holzman C, Wang J, et al. Late-preterm birth and its association with cognitive and socioemotional outcomes at 6 years of age. Pediatrics 2010;126:1124-31.

6. Feldman R. Parent-infant synchrony and the construction of shared timing; physiological precursors, developmental outcomes, and risk conditions. J Child Psychol Psychiatry 2007;48:329-54.

7. Feldman R, Eidelman Al. Neonatal state organization, neuromaturation, mother-infant interaction, and cognitive development in small-for-gestational-age premature infants. Pediatrics 2006;118:e869-78.

8. Greenberg MT, Crnic KA. Longitudinal predictors of developmental status and social interaction in premature and full-term infants at age two. Child Dev 1988;59:554-70.

9. Minde K. Prematurity and serious medical conditions in infancy: Implications for development, behaviour, and intervention. In: Zeanah $\mathrm{CH}$, ed. Handbook of infant mental health. New York: Guilford, 2000:176-94.

10. Treyvaud K, Inder TE, Lee KJ, et al. Can the home environment promote resilience for children born very preterm in the context of social and medical risk? J Exp Child Psychol 2012;112:326-37.

11. Maroney DI. Recognizing the potential effect of stress and trauma on premature infants in the nicu: how are outcomes affected? J Perinatol 2003;23:679-83.
12. Montirosso R, Provenzi L. Implications of epigenetics and stress regulation on research and developmental care of preterm infants. $J$ Obstet Gynecol Neonatal Nurs 2015;44:174-82.

13. Meaney MJ, Szyf M. Maternal care as a model for experience-dependent chromatin plasticity? Trends Neurosci 2005;28:456-63.

14. Mendelsohn A. Recovering reverie: Using infant observation in interventions with traumatised mothers and their premature babies. Infant Obs 2005;8:195-208.

15. Lavelli M, Fogel A. Interdyad differences in early mother-infant face-to-face communication: real-time dynamics and developmental pathways. Dev Psychol 2013;49:2257-71.

16. Lupien SJ, McEwen BS, Gunnar MR, et al. Effects of stress throughout the lifespan on the brain, behaviour and cognition. Nat Rev Neurosci 2009;10:434-45.

17. Bruschweiler-Stern N. Pregnancy: preparation for your new identity. In: Stern D, Bruschweiler-Stern N, eds. The birth of a mother: how the motherhood experience changes you forever. New York: Basic Books, 1998. pp. 31-55.

18. Pancer SM, Pratt M, Hunsberger B, et al. Thinking ahead: complexity of expectations and the transition to parenthood. $J$ Pers 2000;68:253-80.

19. Stern M, Karraker $\mathrm{KH}$, Sopko AM, et al. The prematurity stereotype revisited: impact on mothers' interactions with premature and full-term infants. Infant Ment Health J 2000;21:495-509.

20. Jotzo M, Poets CF. Helping parents cope with the trauma of premature birth: an evaluation of a trauma-preventive psychological intervention. Pediatrics 2005;115:915-19.

21. Stefana A, Lavelli M. I genitori dei bambini prematuri. Una prospettiva psicodinamica. Medico e Bambino 2016;35:327-32.

22. Goldberg S, DiVitto B. Parenting children born preterm. In: Bornstein $\mathrm{M}$, ed. Handbook of Parenting. Mahwah, NJ: Lawrence Erlbaum Associates; 1995:209-231.

23. Siegel LS. Reproductive, perinatal, and environmental factors as predictors of the cognitive and language development of preterm and full-term infants. Child Dev 1982;53:963-73.

24. Lindberg B, Axelsson K, Öhrling K. The birth of premature infants: experiences from the fathers' perspective. J Neonatal Nurs 2007;13:142-9.

25. Pohlman S. Fathering premature infants and the technological imperative of the neonatal intensive care unit: An interpretive inquiry. ANS Adv Nurs Sci 2009;32:E1-16.

26. Fegran L, Helseth S, Fagermoen MS. A comparison of mothers' and fathers' experiences of the attachment process in a neonatal intensive care unit. J Clin Nurs 2008;17:810-16.

27. Lundqvist $P$, Westas $L$, Hallström I. From distance toward proximity: fathers lived experience of caring for their preterm infants. $J$ Pediatr Nurs 2007;22:490-7.

28. Carter JD, Mulder RT, Frampton CMA, et al. Infants admitted to a neonatal intensive care unit: parental psychological status at 9 months. Acta Paediatr 2007;96:1286-9.

29. Zelkowitz P, Bardin C, Papageorgiou A. Anxiety affects the relationship between parents and their very low birth weight infants. Infant Ment Health J 2007;28:296-313.

30. Robertson E, Grace S, Wallington T, et al. Antenatal risk factors for postpartum depression: a synthesis of recent literature. Gen Hosp Psychiatry 2004;26:289-95.

31. Stefana A. La Terapia Intensiva Neonatale, uno spazio per i padri? Psicologia Clinica dello Sviluppo 2016;20:485-91.

32. Gutierrez-Galve L, Stein A, Hanington L, et al. Paternal depression in the postnatal period and child development: mediators and moderators. Pediatrics 2015;135:e339-47.

33. Provenzi L, Santoro $E$. The lived experience of fathers of preterm infants in the neonatal intensive care unit: a systematic review of qualitative studies. J Clin Nurs 2015;24:1784-94.

34. Stefana A, Lavelli M. I padri dei bambini nati pretermine: una risorsa su cui investire. Psicologia Clinica dello Sviluppo 2016;20:165-88.

35. Stefana A, Lavelli $M$. What is hindering research on fathers of premature infants? Minerva Pediatr. (In press). 2017. doi:10.23736/ S0026-4946.16.04618-1

36. Als H, Duffy FH, McAnulty GB, et al. Early experience alters brain function and structure. Pediatrics 2004;113:846-57.

37. Feldman R, Weller A, Sirota L, et al. Skin-to-skin contact (Kangaroo care) promotes self-regulation in premature infants: sleep-wake cyclicity, arousal modulation, and sustained exploration. Dev Psychol 2002;38:194-207.

38. Flacking $\mathrm{R}$, Lehtonen $\mathrm{L}$, Thomson $\mathrm{G}$, et al. Closeness and separation in neonatal intensive care. Acta Paediatr 2012;101:1032-7.

39. Welch MG, Firestein MR, Austin J, et al. Family nurture intervention in the neonatal intensive care unit improves social-relatedness, attention, and neurodevelopment of preterm infants at 18 months in 
a randomized controlled trial. $J$ Child Psychol Psychiatry 2015;56:1202-11.

40. Reynolds LC, Duncan MM, Smith GC, et al. Parental presence and holding in the neonatal intensive care unit and associations with early neurobehavior. J Perinatol 2013;33:636-41.

41. Welch MG, Myers MM, Grieve PG, et al. Electroencephalographic activity of preterm infants is increased by Family Nurture Intervention: a randomized controlled trial in the NICU. Clin Neurophysiol 2014;125:675-84.

42. Goulet C, Bell L, St-Cyr D, et al. A concept analysis of parent-infant attachment. J Adv Nurs 1998;28:1071-81.

43. Filippa M, Devouche E, Arioni C, et al. Live maternal speech and singing have beneficial effects on hospitalized preterm infants. Acta Paediatr 2013;102:1017-20.

44. Krueger C, Parker L, Chiu SH, et al. Maternal voice and short-term outcomes in preterm infants. Dev Psychobiol 2010;52:205-12.

45. Lee $H$, White-Traut $R$. Physiologic responses of preterm infants to the male and female voice in the NICU. J Pediatr Nurs 2014;29:e3-5.

46. Caskey M, Stephens B, Tucker R, et al. Importance of parent talk on the development of preterm infant vocalizations. Pediatrics 2011:128:910-16.

47. Coppola G, Cassibba R. Mothers' social behaviours in the NICU during newborns' hospitalisation: an observational approach. J Reprod Infant Psychol 2010;28:200-11.

48. Feldman R, Eidelman Al. Maternal postpartum behavior and the emergence of infant-mother and infant-father synchrony in preterm and full-term infants: the role of neonatal vagal tone. Dev Psychobiol 2007;49:290-302.

49. Keren M, Feldman R, Eidelman Al, et al. Clinical Interview for high-risk parents of premature infants (CLIP) as a predictor of early disruptions in the mother-infant relationship at the nursery. Infant Ment Health J 2003;24:93-110.

50. Welch MG, Hofer MA, Brunelli SA, et al. Family nurture intervention (FNI): methods and treatment protocol of a randomized controlled trial in the NICU. BMC Pediatr 2012;12:14.

51. Lefkowitz DS, Baxt C, Evans JR. Prevalence and correlates of posttraumatic stress and postpartum depression in parents of infants in the neonatal intensive care unit (NICU). J Clin Psychol Med Settings 2010;17:230-7.

52. Davis L, Edwards $\mathrm{H}$, Mohay $\mathrm{H}$, et al. The impact of very premature birth on the psychological health of mothers. Early Hum Dev 2003;73:61-70.

53. Muller-Nix C, Forcada-Guex M, Pierrehumbert B, et al Prematurity, maternal stress and mother-child interactions. Early Hum Dev 2004;79:145-58.

54. Treyvaud K, Anderson VA, Howard K, et al. Parenting behavior is associated with the early neurobehavioral development of very preterm children. Pediatrics 2009;123:555-61.

55. Treyvaud K, Anderson VA, Lee KJ, et al. Parental mental health and early social-emotional development of children born very preterm. J Pediatr Psychol 2010;35:768-77.

56. Montirosso R, Borgatti R, Trojan S, et al. A comparison of dyadic interactions and coping with still-face in healthy pre-term and full-term infants. Br J Dev Psychol 2010;28(Pt 2):347-68.

57. Forcada-Guex M, Borghini A, Pierrehumbert B, et al. Prematurity, maternal posttraumatic stress and consequences on the mother-infant relationship. Early Hum Dev 2011;87:21-6.

58. Sansavini A, Zavagli V, Guarini A, et al. Dyadic co-regulation, affective intensity and infant's development at 12 months: A comparison among extremely preterm and full-term dyads. Infant Behav Dev 2015;40:29-40.

59. McCormick MC, Workman-Daniels K, Brooks-Gunn J. The behavioral and emotional well-being of school-age children with different birth weights. Pediatrics 1996;97:18-25.

60. Forcada-Guex M, Pierrehumbert B, Borghini A, et al. Early dyadic patterns of mother-infant interactions and outcomes of prematurity at 18 months. Pediatrics 2006;118:e107-14.

61. Korja R, Latva R, Lehtonen L. The effects of preterm birth on mother-infant interaction and attachment during the infant's first two years. Acta Obstet Gynecol Scand 2012;91:164-73.

62. Borghini A, Habersaat S, Forcada-Guex M, et al. Effects of an early intervention on maternal post-traumatic stress symptoms and the quality of mother-infant interaction: the case of preterm birth. Infant Behav Dev 2014;37:624-31.

63. Beebe B, Steele M, Jaffe J, et al. Maternal anxiety symptoms and mother-infant self- and interactive contingency. Infant Ment Health J 2011;32:174-206.

64. Fogel A. Co-regulation, perception and action. Hum Mov Sci 1992;11:505-23.

65. Beebe B, Myers MM, Andrews $\mathrm{H}$, et al. NICU Intervention in First Month Improves Repair of Negative Engagement and Helps Sustain Positive Engagement in Mothers \& Preterm Infants at 4 Months. The New York State Psychiatric Institute, Columbia University Medical Center; International Conference Infant Studies New Orleans, 2016.

66. Flacking R, Dykes F. "Being in a womb" or "playing musical chairs": the impact of place and space on infant feeding in NICUs. BMC Pregnancy Childbirth 2013:13:179.

67. Dykes F, Flacking R. Ethnographic research in maternal and child health. London: Routledge, 2015

68. Hammersley M, Atkinson P. Ethnography: principles in practice. 3rd edn. London: Routledge, 2007.

69. Zuccarini M, Sansavini A, Iverson JM, et al. Object engagement and manipulation in extremely preterm and full term infants at 6 months of age. Res Dev Disabil 2016;55:173-84.

70. Coppola G, Cassibba R, Bosco A, et al. In search of social support in the NICU: features, benefits and antecedents of parents' tendency to share with others the premature birth of their baby. J Matern Fetal Neonatal Med 2013;26:1737-41.

71. Tracey N. Parents of Premature Infants. Their Emotional World. London: Wiley and Sons, 2000.

72. Provenzi L, Barello S, Fumagalli M, et al. A comparison of maternal and paternal experiences of becoming parents of a very preterm infant. J Obstet Gynecol Neonatal Nurs 2016;45:528-41.

73. Arockiasamy V, Holsti L, Albersheim S. Fathers' experiences in the neonatal intensive care unit: a search for control. Pediatrics 2008;121:e215-22.

74. Pohlman S. The primacy of work and fathering preterm infants. Adv Neonatal Care 2005;5:204-16.

75. Arnold L, Sawyer A, Rabe H, et al. Parents' first moments with their very preterm babies: a qualitative study. BMJ Open 2013;3:pii: e002487.

76. Radloff LS. The CES-D Scale: a self-report depression scale for research in the general population. Appl Psychol Meas 1977:1:385-401.

77. Spanier GB. Measuring dyadic adjustment: new scales for assessing the quality of marriage and similar dyads. J Marriage Fam 1976;38:15

78. Scheiner AP, Sexton ME. Prediction of developmental outcome using a perinatal risk inventory. Pediatrics 1991;88:1135-43.

79. Tronick E, Weinberg M. Infant Regulatory Scoring System (IRSS). Boston: The Child Development Unit, Children's Hospital, 1990.

80. Cohn JF, Tronick EZ. Mother-infant face-to-face interaction: influence is bidirectional and unrelated to periodic cycles in either partner's behavior. Dev Psychol 1988;24:386-92.

81. Beebe B, Jaffe J, Markese S, et al. The origins of 12-month attachment: a microanalysis of 4-month mother-infant interaction. Attach Hum Dev 2010;12:3-141.

82. Lavelli M, Beebe B. Coding parent-infant interaction in the NICU. Unpubl Manuscr, 2016.

83. Singer JD, Willett JB. Applied longitudinal data analysis. Oxford: Oxford University Press, 2003

84. Bakeman R, Quera V. Analyzing interaction: sequential analysis with SDIS \& GSEQ. New York: Cambridge University Press, 1995.

85. Moscardino U, Axia G, Scrimin S, et al. Narratives from caregivers of children surviving the terrorist attack in Beslan: issues of health, culture, and resilience. Soc Sci Med 2007;64:1776-87.

86. Lavelli M, Do ge P, Bighin M. Socialization goals of immigrant mothers from diverse cultures and of their childrens preschool teachers in Italy. J Cross Cult Psychol 2016;47:197-214.

87. Braun V, Clarke V. Using thematic analysis in psychology. Qual Res Psychol 2006;3:77-101.

88. Boyatzis R. Transforming qualitative information: thematic analysis and code development. Thousand Oaks: Sage, 1998. 